\title{
SINGULAR PERTURBATIONS
}

BY A. ERDÉLYI ${ }^{1}$

Communicated February 7, 1962

Let $P_{\mathbf{c}}$ designate the problem of finding a solution of the differential equation

$$
\epsilon y^{\prime \prime}+F\left(t, y, y^{\prime}, \epsilon\right)=0, \quad 0 \leqq t \leqq 1,
$$

that satisfies the boundary conditions

$$
y(0)=\alpha(\epsilon), \quad y(1)=\beta(\epsilon) .
$$

Here $\epsilon$ is a small positive parameter approaching zero. We envisage circumstances under which $y=y(t, \epsilon)$ approaches a limit nonuniformly in $t$ as $\epsilon \rightarrow 0+$, the nonuniformity occurring at $t=0$. Accordingly, the limiting problem $P_{0}$ involves the differential equation

$$
F\left(t, u, u^{\prime}, 0\right)=0, \quad 0 \leqq t \leqq 1,
$$

with the single boundary condition

$$
u(1)=\beta(0) \text {. }
$$

Partial derivatives will be denoted by subscripts, thus $F_{\nu}=\partial F / \partial y$, etc.

For a solution $u=u(t)$ of (3) we define the function $\phi$ and the region $D_{\delta}$ by

$$
\begin{aligned}
\phi(t)= & \int_{0}^{t} F_{y^{\prime}}\left(\tau, u(\tau), u^{\prime}(\tau), 0\right) d \tau, \\
D_{\delta}= & {\left[\left(t, y, y^{\prime}, \epsilon\right): 0 \leqq\right.} \\
& t \leqq 1,|y-u(t)|<\delta, \\
& \left.\left|y^{\prime}-u^{\prime}(t)\right|<\delta\left(1+\epsilon^{-1} e^{-\phi(t) / \epsilon}\right), 0<\epsilon<\epsilon_{0}\right] .
\end{aligned}
$$

Assumptions. (A) The problem $P_{0},(3)$ and (4), possesses a solution $u$ which is twice continuously differentiable on $[0,1]$.

(B) For some $\delta>0, F$ possesses partial derivatives of the first and second orders with respect to $y$ and $y^{\prime}$ in $D_{\delta}$, and $F$ as well as these partial derivatives are continuous functions of $t, y, y^{\prime}$ (for fixed $\epsilon$ ).

(C) $F\left(t, u(t), u^{\prime}(t), \epsilon\right)=O(\epsilon) ; q(t, \epsilon)=F_{y}\left(t, u(t), u^{\prime}(t), \epsilon\right)=O(1)$; $p(t, \epsilon)=F_{y^{\prime}}\left(t, u(t), u^{\prime}(t), \epsilon\right)=\phi^{\prime}(t)+\epsilon p_{1}(t, \epsilon)$ where $\phi$ is twice continu-

1 This research was supported in part by the National Science Foundation under Grant No. NSF G-19914. 
ously differentiable in $[0,1], \phi(0)=0, \phi^{\prime}(t)>0, p_{1}$ is a continuous function of $t$ and $p_{1}(t, \epsilon)=O(1) ; F_{y y}\left(t, y, y^{\prime}, \epsilon\right)=O(1), F_{y y^{\prime}}\left(t, y, y^{\prime}, \epsilon\right)=O(1)$, $F_{y^{\prime} y^{\prime}}\left(t, y, y^{\prime}, \epsilon\right)=O(\epsilon)$. All order relations (here and later) hold as $\epsilon \rightarrow 0+$, uniformly in all the other variables in their respective ranges.

(D) $\beta(\epsilon)-\beta(0)=O(\epsilon)$.

(E) $F_{y}\left(t, y, y^{\prime}, \epsilon\right)=O(1), F_{y^{\prime}}\left(t, y, y^{\prime}, \epsilon\right) \geqq B>0$ in $D_{\delta}$.

Coddington and Levinson [1] discussed the problem $P_{\mathrm{e}}$ under the assumption that $F$ is linear in $y^{\prime}$ (and, for the sake of simplicity, $F$, $\alpha$, and $\beta$ are independent of $\epsilon$ ). Their differentiability conditions on $F$ were somewhat milder than ours, and they proved that $y(t, \epsilon)$ $\rightarrow u(t)$ and $y^{\prime}(t, \epsilon) \rightarrow u^{\prime}(t)$ as $\epsilon \rightarrow 0+$, uniformly on any interval $0<\delta \leqq t \leqq 1$. Wasow [2] also assumed $F$ to be linear in $y^{\prime}$ and in addition he assumed $F$ to be analytic in $y$ and $\epsilon$. He established results, including asymptotic expansions, valid throughout $[0,1]$. Briš $[3]$ considered $F$ that are nonlinear in $y^{\prime}$ and independent of $\epsilon$ : his conditions neither include ours, nor are they included by ours.

Theorem. Under assumptions (A) to (D) there exists a $\mu_{0}>0$, independent of $\epsilon$, so that whenever $|\alpha(\epsilon)-u(0)|<\mu_{0}$, the boundary value problem $P_{\epsilon}$ possesses a solution $y=y(t, \epsilon)$ for each sufficiently small $\epsilon$. Moreover, $y=u+v+w$, where $y^{*}=u+v$ satisfies (1) and the second boundary condition (2), $v(t), v^{\prime}(t)=O(\epsilon)$, and $w(t), \epsilon w^{\prime}(t)=O(\exp [-\phi(t) / \epsilon])$. Under the further assumption (E), $y$ is the only solution of $P_{\mathrm{\epsilon}}$ in $D_{\delta}$.

The proof of this result follows the pattern set by Wasow in that first $v$ is constructed by "linearizing" the differential equation "around $u$," and then $w$ is constructed by linearizing around $y^{*}=u+v$. The technique employed in carrying out these steps differs from Wasow's. While Wasow employs power series-asymptotic expansions combined with integral equations in the case of $v$, and convergent power series expansions in the case of $w$, all constructions here are based directly on integral equations. This procedure makes the analyticity assumptions on $F$ unnecessary and makes it easier to include differential equations which are not linear in $y^{\prime}$.

First the linear differential equation

$$
\epsilon V^{\prime \prime}+p(t, \epsilon) V^{\prime}+q(t, \epsilon) V=0
$$

is investigated: $p$ and $q$ are subject to assumptions obtained from (B) and (C) if one sets $F=p y^{\prime}+q y$. The results are known in essence from the asymptotic theory of linear differential equations. They are obtained here, under milder differentiability conditions than is usual, from the integral equation 
(6) $\quad V(t)=1+\int_{0}^{1}\left(1-e^{\theta(\tau, 1)}\right) \bar{V}(\tau) d \tau-\int_{0}^{t}\left(1-e^{\theta(\tau, t)}\right) \bar{V}(\tau) d \tau$, where

$$
\begin{aligned}
\theta(s, t) & =[\phi(s)-\phi(t)] / \epsilon, \\
\bar{V}(t) \phi^{\prime}(t) & =\left[p(t, \epsilon)-\phi^{\prime}(t)+\epsilon \phi^{\prime \prime}(t) / \phi^{\prime}(t)\right] V^{\prime}(t)+q(t, \epsilon) V(t) .
\end{aligned}
$$

The principal results on (6) state the existence of two solutions $V_{1}$ and $V_{2}$ such that

$$
\begin{aligned}
& V_{1}(1)=1, \quad V_{1}^{\prime}(0)=0, \quad V_{1}(t)=e^{\psi(1)-\psi(t)}+O(\epsilon), \\
& V_{1}^{\prime}(t)=-\psi^{\prime}(t) e^{\psi(1)-\psi(t)}\left[1-e^{-\phi(t) / \epsilon}\right]+o(1), \\
& V_{2}(0)=1, \quad V_{2}(t)=e^{\psi_{2}(t)-\phi(t) / \epsilon}+O\left(\epsilon e^{-\phi(t) / \epsilon}\right), \\
& V_{2}^{\prime}(t)=-\epsilon^{-1} \phi^{\prime}(t) e^{\psi_{2}(t)-\phi(t) / \epsilon}+O\left(e^{-\phi(t) / \epsilon}\right),
\end{aligned}
$$

where

$$
\begin{aligned}
\psi(t) & =\psi(t, \epsilon)=\int_{0}^{t} \frac{q(\tau, \epsilon)}{\phi^{\prime}(\tau)} d \tau, \\
\psi_{2}(t, \epsilon) & =\psi(t, \epsilon)-\int_{0}^{t} P_{2}(t, \epsilon) d t .
\end{aligned}
$$

$V_{1}$ is the solution of (6), and $V_{2}$ is obtained by a transformation. Asymptotic forms of other solutions of (5) follow readily from (7) and (8).

We now set $y^{*}=u+v$ in (1) and rewrite this differential equation in the form

$$
\epsilon v^{\prime \prime}+p(t, \epsilon) v^{\prime}+q(t, \epsilon) v=G\left(t, v, v^{\prime}, \epsilon\right),
$$

where $p$ and $q$ are as in assumption (C). A solution of (9) satisfying $v(1)=\beta(\epsilon)-\beta(0)$ is obtained as a solution, by successive approximations, of the integral equation

$$
\begin{aligned}
v(t)= & {\left[\beta(\epsilon)-\beta(0)-\int_{0}^{1} K(1, s) G\left(s, v(s), v^{\prime}(s), \epsilon\right) d s\right] V_{1}(t) } \\
& +\int_{0}^{t} K(t, s) G\left(s, v(s), v^{\prime}(s), \epsilon\right) d s,
\end{aligned}
$$


where

$$
K(t, s)=\frac{1}{\epsilon} \frac{V_{1}(s) V_{2}(t)-V_{1}(t) V_{2}(s)}{V_{1}(s) V_{2}^{\prime}(s)-V_{1}^{\prime}(s) V_{2}(s)}
$$

and this solution is shown to possess the properties asserted in the theorem.

Setting $y=y^{*}+w=u+v+w$ in (1), we see that the boundary layer correction $w$ satisfies a differential equation that can be written as

$$
\epsilon w^{\prime \prime}+p^{*}(t, \epsilon) w^{\prime}+q^{*}(t, \epsilon) w=G^{*}\left(t, w, w^{\prime}, \epsilon\right),
$$

where

$$
p^{*}(t, \epsilon)=F_{y^{\prime}}\left(t, y^{*}(t), y^{* \prime}(t), \epsilon\right), \quad q^{*}(t, \epsilon)=F_{y}\left(t, y^{*}(t), y^{* \prime}(t), \epsilon\right) .
$$

$w$ also satisfies the boundary conditions

$$
w(0)=\alpha(\epsilon)-u(0)-v(0)=\mu, w(1)=0 .
$$

The linear part of (11) is again of the form (5), with $p$ and $q$ replaced by $p^{*}$ and $q^{*}$. Constructing $K^{*}$ as before, and setting

$$
V_{3}^{*}(t)=\frac{V_{1}^{*}(t) V_{2}^{*}(1)-V_{1}^{*}(1) V_{2}^{*}(t)}{V_{1}^{*}(0) V_{2}^{*}(1)-V_{1}^{*}(1) V_{2}^{*}(0)},
$$

we see that $w$ satisfies the integral equation

$$
\begin{aligned}
w(t)= & {\left[\mu+\int_{0}^{1} K^{*}(0, s) G^{*}\left(s, w(s), w^{\prime}(s), \epsilon\right) d s\right] V_{3}^{*}(t) } \\
& -\int_{t}^{1} K^{*}(t, s) G^{*}\left(s, w(s), w^{\prime}(s), \epsilon\right) d s .
\end{aligned}
$$

Again the integral equation can be solved by successive approximations and those properties of $w$ asserted in the theorem follow.

Lastly, the uniqueness of the solution of $P_{\epsilon}$ is established as follows. That solution, $y=y(t, \epsilon, \gamma)$ of (1) satisfying the initial conditions

$$
y(0)=\alpha(\epsilon), \quad y^{\prime}(0)=\gamma
$$

is unique as far as it remains in $D_{\delta}$, and this solution is differentiable with respect to $\gamma \cdot z=\partial y / \partial \gamma$ satisfies the "variational equation"

$$
\epsilon z^{\prime \prime}+F_{y^{\prime}}\left(t, y(t), y^{\prime}(t), \epsilon\right) z^{\prime}+F_{y}\left(t, y(t), y^{\prime}(t), \epsilon\right) z=0
$$

and the initial conditions $z(0)=0, z^{\prime}(0)=1$. Now, (13) is again of the form (5) and it can be shown that $z(1)>0$. Thus, $y(1, \epsilon, \gamma)$ is a strictly 
increasing function of $\gamma$, and for a fixed sufficiently small $\epsilon$ there is at most one $\gamma=\gamma(\epsilon)$ so that $y(1, \epsilon, \gamma(\epsilon))=\beta(\epsilon)$.

A detailed presentation of this result together with some further developments will appear elsewhere.

\section{REFERENCES}

1. E. A. Coddington and N. Levinson, $A$ boundary value problem for a non-linear differential equation with a small parameter, Proc. Amer. Math. Soc. 3 (1952), 73-81.

2. W. Wasow, Singular perturbations of boundary value problems for nonlinear differential equations of the second order, Comm. Pure Appl. Math. 9 (1956), 93-113.

3. N. I. Briš, On boundary problems for the equation $\epsilon y^{\prime \prime}=f\left(x, y, y^{\prime}, \epsilon\right)$ for small $\epsilon^{\prime} s$, Dokl. Akad. Nauk SSSR (N.S.) 95 (1954), 429-432; M.R. 16, 251.

California Institute of Technology 